\title{
Cortisol metabolism during acute stress-an (IC)U turn
}

A new study in critically ill patients shows that reduced cortisol clearance, rather than increased cortisol production, is the main driving force behind hypercortisolaemia in these patients.

During critical illness, plasma cortisol levels are elevated, which was thought to be due to activation of the hypothalamicpituitary-adrenal (HPA) axis, with an increased release of adrenocorticotropic hormone (ACTH) by the pituitary gland causing an increase in cortisol production. However, "data on plasma ACTH concentrations during critical illness were scarce and low values have been reported," says lead researcher Greet Van den Berghe (Catholic University, Leuven, Belgium). "This raised the hypothesis that the classical concept of an activated HPA axis during critical illness may be wrong."

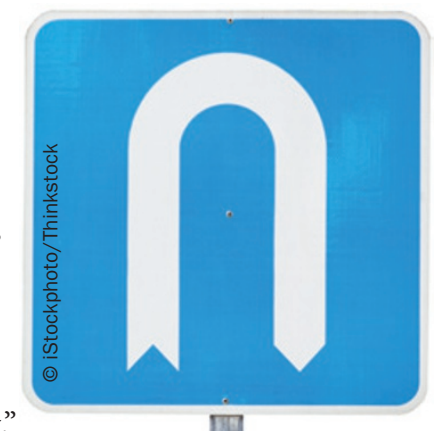

production, and the expression and activity of cortisol-metabolizing enzymes in 158 patients treated at an intensive care unit (ICU) and 64 healthy controls. Cortisol levels in patients were 3.5-fold higher than in controls. Cortisol production in patients was less than twice that seen in controls, but cortisol clearance was reduced by $>50 \%$. Of note, patients' cortisol levels did not correlate with ACTH levels. However, reduced cortisol clearance was associated with reduced expression and activity of cortisol-metabolizing enzymes in the liver and kidney, "possibly evoked by bile acids," comments Van den Berghe.

The researchers plan to investigate the effect of reduced cortisol metabolism in different tissues of the body, the role of bile acids in this process and the consequences of

The reseachers assessed plasma levels of ACTH and cortisol, cortisol clearance and long-term ACTH suppression in ICU patients. "The dose of hydrocortisone of
200 mg per day used to treat ICU patients with suspected adrenal failure seems to be threefold to sixfold too high: this should be further investigated in randomized controlled trials," says Van den Berghe.

"The diagnosis of relative adrenal insufficiency during long-term stress should be totally revised," comment Roger Bouillon (also from Catholic University, Leuven) and Steven Lamberts (Erasmus Medical Centre, Rotterdam, The Netherlands), who were not authors of the study. More generally, "assessment of adrenal function should be based on hormone production and clearance, free hormone availability at target-tissue level (taking into account local concentrations of binding proteins) and hormone action," add Bouillon and Lamberts.

Joana Osório

Original article Boonen, E. et al. Reduced cortisol metabolism during critical illness. N. Engl. J. Med. doi:10.1056/NEJMoa1214969 